\title{
HD Term Type
}

National Cancer Institute

\section{Source}

National Cancer Institute. HD Term Type. NCI Thesaurus. Code C45776.

The $\mathrm{NCl}$ term type designation for a header term. 